\title{
Structured Student-Generated Videos for First-Year Students at a Dental School in Malaysia
}

\author{
Hanan Omar, B.D.S., M.Sc., Ph.D.; Saad A. Khan, B.D.S., M.Sc.; \\ Chooi G. Toh, B.D.S. (Hons), M.Sc., F.D.S.R.C.P.S., D.R.D.R.C.S., F.D.S.R.C.S.
}

Abstract: Student-generated videos provide an authentic learning experience for students, enhance motivation and engagement, improve communication skills, and improve collaborative learning skills. This article describes the development and implementation of a student-generated video activity as part of a knowledge, observation, simulation, and experience (KOSE) program at the School of Dentistry, International Medical University, Kuala Lumpur, Malaysia. It also reports the students' perceptions of an activity that introduced first-year dental students $(n=44)$ to clinical scenarios involving patients and dental team aiming to improve professional behavior and communication skills. The learning activity was divided into three phases: preparatory phase, video production phase, and video-watching. Students were organized into five groups and were instructed to generate videos addressing given clinical scenarios. Following the activity, students' perceptions were assessed with a questionnaire. The results showed that 86 percent and 88 percent, respectively, of the students agreed that preparation of the activity enhanced their understanding of the role of dentists in provision of health care and the role of enhanced teamwork. In addition, 86 percent and 75 percent, respectively, agreed that the activity improved their communication and project management skills. Overall, the dental students perceived that the student-generated video activity was a positive experience and enabled them to play the major role in driving their learning process.

Dr. Omar is Associate Professor, School of Dentistry, International Medical University, Kuala Lumpur, Malaysia; Dr. Khan is a Ph.D. scholar, Department of Conservative Dentistry, Faculty of Dentistry, University of Malaya, Kuala Lumpur, Malaysia; and Dr. Toh is Professor, School of Dentistry, International Medical University, Kuala Lumpur, Malaysia. Direct correspondence and requests for reprints to Dr. Hanan Omar, International Medical University, No. 126, Jalan Jalil Perkasa 19, Bukit Jalil, Kuala Lumpur, 57000, Malaysia; hanan_omar@imu.edu.my.

Keywords: educational technologies, video production, communication skills, professionalism, dental students, Malaysia

Submitted for publication 2/18/12; accepted 7/11/12

$\mathrm{T}$ The aim of undergraduate dental education has been defined by the United Kingdom's General Dental Council as producing "a caring, knowledgeable, competent, and skillful dentist. Dental graduates should be able to accept professional responsibility for the effective and safe care of patients. This can be achieved through appreciating the need for continuing professional development, in addition to utilizing advances in relevant knowledge and techniques, and understanding the role of the patient in decision making." "Outcomes-based education is an approach that shifts emphasis from what the teacher will teach to what the student can perform. It encourages student learning by clear and explicit identification of what a learner is expected to know, comprehend, and able to do along with teaching and learning activities that elicit the specified outcomes. ${ }^{2}$

Dental education aims to prepare graduates to treat the patient holistically and to meet the professional competencies suitable for lifetime practice. Personal and professional development (PPD) is an integral part of dental education that aims to prepare those graduates for a life of continuous learning. Hence, the development of a foundation for theory and practice of PPD is considered a pressing need for contemporary dental education. Lifelong learning skills and cultivated habits, professional ethics, and moral responsibility as part of PPD need to be included in dental education outcomes and can be supported by new learning activities such as using electronic media and clinical simulation. ${ }^{3}$

The use of student-generated video as a learning tool in higher education contexts was described by $\mathrm{Crean}^{4}$ and Ludewig ${ }^{5}$ in 2001 . The rationale for using student-generated videos includes provision of authentic learning experience, enhancement of motivation and engagement, improvement of communication skills, and improvement in collaborative learning skills. ${ }^{6,7}$ Student-generated videos have also been described as a potential tool to achieve cross-curricular outcomes and to facilitate multidisciplinary learning. ${ }^{8}$

The use of student-generated video as an educational method relies on a range of theories. ${ }^{9,10}$ 
The theory used in our study is experiential learning theory, which is important in acquisition of skills through four learning environments: affectively oriented (feeling), symbolically oriented (thinking), perceptually oriented (watching), and behaviorally oriented (doing). In each of these four environments, there are two tasks. First is "grasping," which consists of concrete experiences and abstract conceptualization. Second is "transforming," which consists of reflection and action. Well-structured student-generated videos with feedback encourage learners to use all four environments and to complete both tasks of each environment. ${ }^{9}$ Student-generated videos also address the principles associated with adult learning described by Knowles et al., including the need of the learner to know, the learner being self-directed, problemcentered learning, and internal motivation to learn. ${ }^{10}$

Roleplays and clinical simulations are commonly used in medical and dental education as training methods to help students develop knowledge, attitudes, and skills in a range of disciplines. In dental education, they can provide early exposure of dental students to clinical situations and help to familiarize them with the dental setup. The use of roleplays was described in dentistry as early as 1974 by Gershen and Handelman. ${ }^{11}$ In 2005, Kneebone described a theory-based approach to learning clinical skills in simulation: simulation as a training method included sustained and deliberate practice in a safe environment, accessibility to expert facilitators who are available only when needed, introducing students to real-life clinical situations, and providing them with supportive, motivational, and learner-centered environments. ${ }^{12}$ Although these advantages of simulation were defined in relation to practicing clinical procedures, the benefits of simulation can extend to improving communication and other related professional skills. The aim of this study was to describe the development and implementation of a structured student-generated videos program in a dental school in Malaysia and to assess the students' perception of the program.

\section{Materials and Methods}

The aim of the program was to introduce firstyear dental students to clinical scenarios with patient and dental team involvement to improve professional behavior towards patients and dental team in addition to communication skills. The development of the program was based on Miller's framework for clini- cal competence, ${ }^{13}$ and the program was comprised of Miller's four components: knowledge, observation, simulation, and experience (KOSE). Knowledge was acquired through lectures and problem-based learning covering the learning outcomes. Students were introduced to dental clinic activities through clinical sessions and visits to dental clinics and dental laboratories to observe various clinical/laboratory activities and situations. Simulation was achieved through the production of videos, and experience was obtained during the clinical work represented by treating and managing patients in the dental setup.

A two-hour lecture was delivered to first-year dental students addressing communication skills (knowledge). Then, a total of nine hours of visits to dental clinics and dental laboratories were scheduled for students to observe the workflow and conduct of various clinical and laboratory activities respectively (observation). For the simulation, interactive clinical sessions were held in which the students were divided into groups of three with one student portraying a patient, one student roleplaying the clinician, and one student as the assistant. The students were required to conduct various tasks during these clinical sessions such as taking medical and dental history and other clinical activities such as dental charting, periodontal charting, and scaling and polishing, with close support provided by the assistant.

The second part of the simulation involved the production of student-generated videos, which is the focus of this study. Student-generated videos formed part of the second semester curriculum activities, which included a comprehensive coverage of patient care including interaction of the dental team, protection of patients' rights, confidentiality, and keeping of accurate dental records. Learning outcomes for the student video project are shown in Table 1. The learning activities were organized into three phases (Table 2).

Phase I was the preparatory phase by faculty. Five short scenarios were developed by two faculty members to address the learning outcomes of the program. A briefing manual was developed to guide the students to achieve the expected outcomes of the activity. The manual included grouping the students and scenario assigned for each group, the developed scenarios, description of the expected roleplay and timeline of the activity, and instructions on generating the videos. The subject of the scenarios and the learning outcomes for each are shown in Table 3.

Phase II was development of the student-generated videos. First-year dental students in their second 


\section{Table 1. Learning objectives of the KOSE program and the student-generated videos}

Learning Objectives

1. Appreciate the importance of professional behavior in the dental clinic.

2. Appreciate the dynamics of patient-dentist relationship.

3. Appreciate the collaborative teamwork of a team in the practice and the implications of behavior in the delivery of oral health care.

4. Understand role, responsibilities, and activities of a dentist in the provision of health care to the community.

5. Understand interprofessional relationships that exist in provision of oral care.

6. Demonstrate appropriate attitudes and ethics required for the compassionate and caring practice of dentistry.

7. Demonstrate commitment to maintaining professional standards and obligations.

8. Demonstrate responsible and professional behavior towards patients.

9. Able to work cooperatively as a member of a team in the practice of dentistry.

10. Able to recognize the physical, emotional, and social needs of patients and respond appropriately with sensitivity.

Table 2. Preparation and implementation of and feedback on the student-generated videos

Phase 1: Preparatory Phase by Faculty

- Five short scripts were developed by faculty members addressing the learning outcomes and representing situations that might occur in a dental setup.

- Preparation of students' briefing manual that included students' grouping, the scenarios, time frame of the activity, and instructions for video production.

Phase 2: Student-Generated Videos

Week 1 One-hour briefing session about the activity: introduction to scenarios

Introduction Independent learning using online resources in the e-learning portal:

- Briefing manual

- Online reading materials

- Links to video production resources

Script preparation and submission, followed by discussion of the submitted scripts

Week $2 \quad$ Video generation by the students.

Phase 3: Video-Watching Sessions

Week 3 Three-hour sessions:

- Generated videos were watched by the students and three faculty members.

- Videos were discussed and evaluated by students and faculty members.

Phase 4: Students' Perceptions of Videos

Week 3 Administration of questionnaire following video-watching sessions.

semester $(\mathrm{N}=44)$ were organized randomly into five groups, and each group was assigned a scenario. Students were instructed to write two scripts for each scenario under the titles of "What went wrong?" and "The way it should be." Scripts were revised and modified by faculty members to ensure that the learning outcomes were covered. Director, actors, and technical crew for each scenario were assigned by the group, ensuring the participation of all students in the process of video generation. Following script approval, the students proceeded to videotape the scripts in two two-minute videos per scenario.
Students were allowed to use any video recording device they had available and to use free software for editing their recorded videos. No formal lessons were provided in video production, and students had to use their own resources to generate the videos. The timeline for video production was three weeks.

Phase III was the video-watching sessions. Students watched the videos in the presence of three faculty members and the entire class of students during two assigned sessions. Feedback on the produced videos was provided by the faculty, addressing the learning outcomes and the videos generated. 
Table 3. Learning outcomes for each scenario

Scenario

Scenario 1: Patient confides to her dentist that she is HIV-positive and is concerned about treatment refusal and cross-infection.

Scenario 2: A close friend who is a patient of the dentist inquires about the dental condition of another patient as he happens to know her. The friend asks both the dentist and the receptionist about this patient.

Scenario 3: A mother and her daughter arrive at the clinic when the dentist is about to leave. The daughter is in pain, and they cannot communicate in English.

Scenario 4: After taking radiographs of the patient, the dentist discovers that, due to faulty processing of films, the images are distorted and there is a need to take a new set of radiographs.

Scenario 5: A removable prosthesis cannot be delivered due to technical problems that might arise in the future, and the patient is leaving for a conference the next day.
Learning Outcomes

1. Identify roles and responsibilities of the dentist toward the patient and patient's rights.

2. Understand dynamic of dentist-patient relationship.

3. Demonstrate ability to explain to the patient about infection control in dentistry and standard precautions for infection control.

1. Identify roles and responsibilities of the dentist and the dental team in ensuring confidentiality of patients.

2. Appreciate the importance of separating personal and professional relations.

1. Handle appointment situations in the dental clinic.

2. Address cultural diversity and language barriers that might be encountered during dental treatment.

1. Understand the importance of informing patients about problems encountered while conducting dental procedures.

2. Identify importance of the dental team and handling the problems as a team.

3. Identify the importance of patient's safety.

1. Handle dentist-technician communication.

2. Involve patients in decision making.

3. Learn to admit mistakes, and demonstrate ability to handle difficult situations.
We identified four areas for evaluation: video production, skills developed during video production, benefits of watching the videos, and general aspects of the activity. Subtopics were identified for the first three parts based on relevance and were included in the questionnaire after consensus was reached among the investigators. The general aspects of the activity part of the questionnaire were adapted from a questionnaire developed to assess communication skills of dental students. ${ }^{14}$

The questionnaire was evaluated by five dental students in a separate cohort and one subject expert and was amended according to their recommendations. The questionnaire was then piloted with another five dental students. Cronbach's alpha coefficient was measured to determine the internal consistency of various sections of the questionnaire. It was found to be 0.843 for the items representing preparation of videos, 0.813 for the items representing the skills developed during the video generation, and 0.789 for items representing the benefits from watching the videos. These values are considered preferable and showed good internal consistency.

The students' perceptions of the video-generation activity was assessed after the second video watching session with a nineteen-item Likert-scale questionnaire addressing preparation, participation, the produced videos, and the learning activity overall.

\section{Results}

The activity involved forty-four first-year dental students in their second semester. Forty-three students participated in the evaluation yielding a response rate of 98 percent. Table 4 shows the distribution of students' perceptions of the activity. Student perceptions were generally positive. Even though there were some disagreements among the items evaluated, the majority of students agreed that they had learned from the activity. The nineteen items of the questionnaire were divided into four sections. Three items pertained to preparation for video production, five dealt with skills developed by the students during participation in the video-generation, six explored the benefits of watching the videos, and the last section addressed general aspects of the learning activity.

In the first section regarding preparation for video production, 84 percent of the responding students agreed on the three items assessed, 7 percent were neutral, and 9 percent disagreed. Seventy-nine 
Table 4. Distribution of students' ratings of the activity

Item $\begin{gathered}\text { Agre } \\ \mathrm{n}(\%)\end{gathered}$

Student Preparation for Video Production

Q1 Preparing the scripts directed me toward reading about the topics involved.

Q2 Preparing the scripts enhanced my understanding of the role of a dentist in serving patients.

Q3 Preparing the scripts directed me to work more closely with my team. Average rating and percentage for section

\begin{tabular}{|c|c|c|}
\hline $34(79 \%)$ & $3(7 \%)$ & $6(14 \%)$ \\
\hline 37 (86\%) & $2(5 \%)$ & $4(9 \%)$ \\
\hline 38 (88\%) & $3(7 \%)$ & $2(5 \%)$ \\
\hline $36(84 \%)$ & $3(7 \%)$ & $4(9 \%)$ \\
\hline 37 (86\%) & $4(9 \%)$ & $2(5 \%)$ \\
\hline $34(79 \%)$ & $6(14 \%)$ & $3(7 \%)$ \\
\hline 31 (72\%) & 7 (16\%) & $5(12 \%)$ \\
\hline $25(58 \%)$ & $8(19 \%)$ & $10(23 \%)$ \\
\hline $34(79 \%)$ & $2(5 \%)$ & $7(16 \%)$ \\
\hline $32(74 \%)$ & $6(14 \%)$ & $5(12 \%)$ \\
\hline 35 (81\%) & $6(14 \%)$ & $2(5 \%)$ \\
\hline $33(77 \%)$ & $7(16 \%)$ & $3(7 \%)$ \\
\hline $30(70 \%)$ & $7(16 \%)$ & $6(14 \%)$ \\
\hline $28(65 \%)$ & $8(19 \%)$ & $7(16 \%)$ \\
\hline $24(55 \%)$ & $10(23 \%)$ & $9(20 \%)$ \\
\hline $40(93 \%)$ & $3(7 \%)$ & \\
\hline 32 (74\%) & $6(14 \%)$ & $5(12 \%)$ \\
\hline 27 (62\%) & $9(21 \%)$ & $7(16 \%)$ \\
\hline 36 (83\%) & $3(7 \%)$ & $4(9 \%)$ \\
\hline $24(56 \%)$ & $2(5 \%)$ & $17(40 \%)$ \\
\hline 39 (91\%) & $4(9 \%)$ & 0 \\
\hline 37 (86\%) & $1(2 \%)$ & $5(12 \%)$ \\
\hline $33(77 \%)$ & $4(9 \%)$ & $7(16 \%)$ \\
\hline
\end{tabular}

Skills Developed in Students During Participation in Video Generation

Q4 Participation in video generation improved my language and communication skills.

Q5 Participation in video generation improved my project management skills.

Q6 Participation in video generation improved my analytical skills.

Q7 Participation in video generation improved my problem-solving skills.

Q8 Participation in video generation improved my organizational skills. Average rating and percentage for section

Benefits of Watching Videos

Q9 Watching the videos enhanced my understanding of the role of the dentist in provision of health care.

Q10 Watching the videos improved my understanding of patients' rights.

Q11 Watching the videos helped me distinguish between less and more effective clinician-patient communication.

Q12 Watching the videos improved my understanding of professional behavior in dental clinic.

Q13 Watching the videos enabled me to understand better the safety precautions in the dental clinic.

Q14 The videos demonstrated responsible and professional behavior towards patients.

Average rating and percentage for section

General Aspects of the Learning Activity

Q15 I have learned about the many subtle factors that influence communication from this activity that I will use in future with patients; these include body language, tone of voice, and speech volume.

Q16 I have learned that the video medium can be a powerful communication tool.

Q17 The right amount of time was allotted for this activity.

Q18 Overall, this activity was worthwhile.

Q19 I would like to participate in this type of activity again.

Average rating and percentage for section

Agree

Neutral n $(\%)$

Disagree n $(\%)$

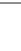


vided a neutral response, and 12 percent disagreed that they benefited from watching the videos. Eightyone percent agreed that the videos enhanced their understanding of the role of dentists in provision of health care. Seventy-seven percent and 65 percent, respectively, agreed that the videos improved their understanding of patients' rights and their understanding of professional behavior in the dental clinic; 70 percent reported the videos were helpful in distinguishing between less and more effective patientdentist communication; and 55 percent agreed that the videos helped them develop better understanding of safety precautions in the dental clinic. Ninety-three percent agreed that the videos helped them demonstrate professional behavior towards their patients.

General aspects of the learning activity were addressed in the last part of the questionnaire. On those questions, 77 percent of the responding students agreed that the experience in general was positive, 9 percent reported the activity was neutral, and 16 percent disagreed that the activity was positive. Sixty-two percent agreed that the activity helped them to learn factors that might affect communication skills, and 83 percent agreed that the video medium could be a powerful communication tool (Figure 1). Regarding the time allotted for the activity, 56 percent agreed that the time was sufficient to prepare the vid- eos. Ninety-one percent agreed that the activity was worthwhile, and 86 percent reported that they would like to participate in similar activities in the future.

\section{Discussion}

The activity described in this study was based on a KOSE program as KOSE has been found to be a successful approach to communication skills training in a dental school. ${ }^{14}$ The student-generated video activity was used to introduce an early appreciation of the clinical role and responsibilities of a dentist in the dental program as part of a vertically integrated dental curriculum.

Another study found that student-generated videos helped in developing an understanding of the outcomes of the activity, increased students' autonomy, promoted active learning, and provided the opportunity for group learning as well as language development. ${ }^{15}$ Enhancing motivation of the students has also been reported to be one of the most important benefits that can be obtained through studentgenerated videos. ${ }^{16}$ In our study, the learning activity developers observed that their role in the activity was directed more towards facilitating the process and providing the students with minimal supervision

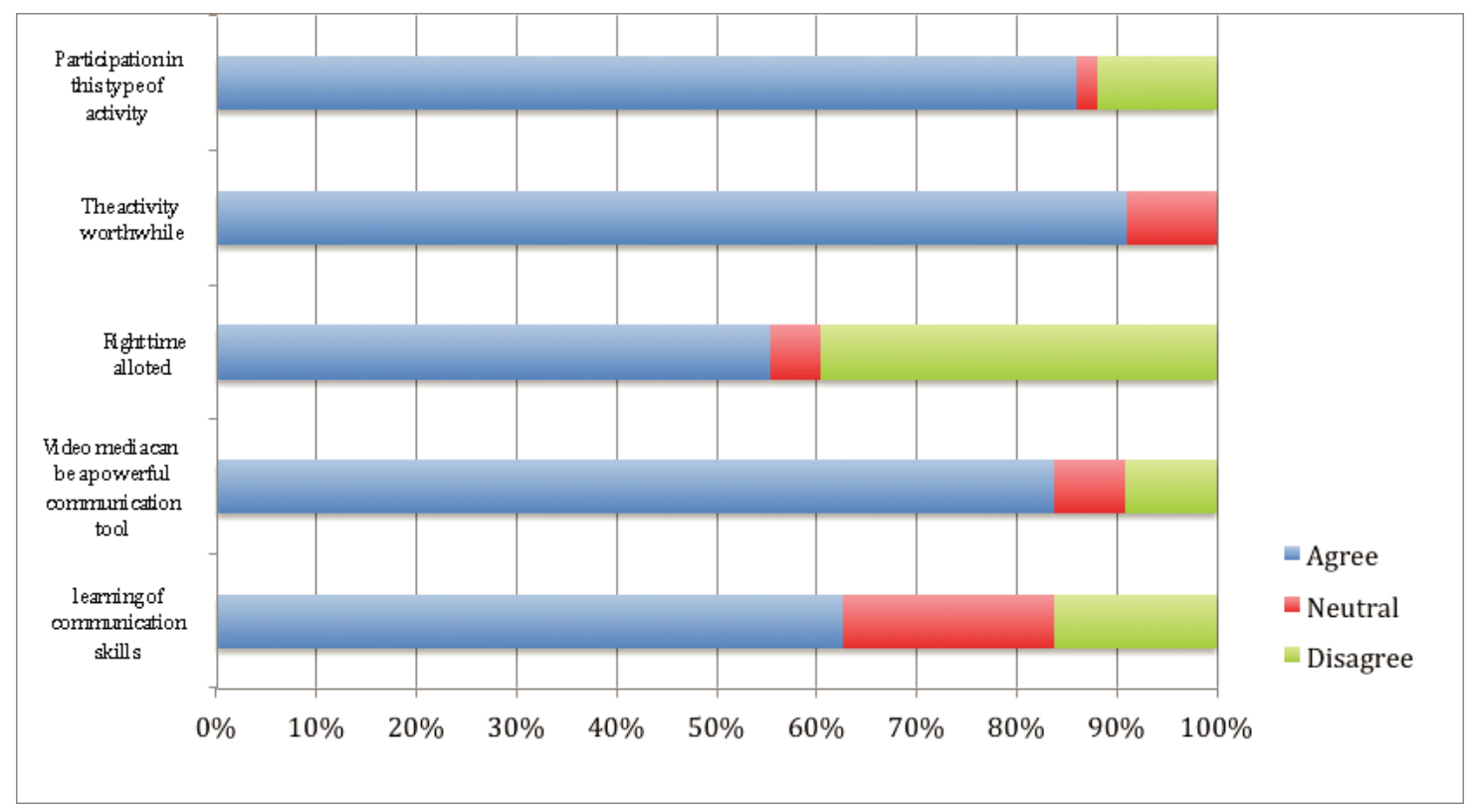

Figure 1. Students' general perceptions of the video activity 
and direction during the production phase. Students were allowed a lot of independence in deciding on the scripting, directing the videos, and identifying the roles they played. Group effort was evident in the videos, which can be attributed to the nature of the activity. The students played the major role in driving the learning process by providing written scripts and leading discussions during the videowatching sessions. The student-generated videos as part of the KOSE program addressed the strategies recommended for communication skills training: small-group experience, performance-based instruction, simulation, skills assessment, and feedback. ${ }^{14}$ Eighty-four percent of the responding students agreed that the activity directed them towards reading, enhanced their understanding of the role of dentists, and helped them to work closely as a team.

In regards to the skills developed in students, 74 percent of the participating students reported improvement in the stated skills. The majority of the students agreed that participation in producing the videos improved their language and communication skills, management skills, analytical skills, and organization skills. The problem-solving skills results were inconclusive and did not demonstrate a significant increase or improvement in the students' perception of this skill: only 58 percent agreed that the generation of videos improved their problemsolving skills. We feel that this reporting can be due to using more than one scenario with various degrees of difficulty in producing the videos.

Seventy-four percent of the participating students agreed that they benefited from watching the videos. They reported that watching the videos enhanced their understanding of the role of the dentist in provision of health care and appreciation of patients' rights and helped improve their ability to distinguish between less and more effective clinical patient communication. These findings can be attributed to the production of two videos on the topics of what went wrong and the way it should be. These videos may have deepened the students' understanding of proper and improper attitudes in providing oral health care and professional behavior. Students' appreciation of safety precautions in the dental clinic was not clearly identified as only 55 percent of the respondents agreed that the videos helped them to understand safety precautions in the clinic. The lack of clinical experience and possibly inadequate dramatization by the group concerned may have contributed to students' lack of appreciation concerning issues related to patient safety.
The last part of the questionnaire addressed the learning activity as a whole, and the majority of students reported appreciation of the video medium as a powerful communication tool and agreed that the activity was worthwhile. They also agreed to participate in a similar type of activity again. However, regarding the time allotted for the activity, 45 percent expressed that the time allotted was not enough. We concluded that the result obtained for this section (with 16 percent disagreeing that the activity as a whole was useful) was mainly attributed to the time allotted to preparing the activity.

Another study placed significant emphasis on the importance of the qualities of student-made videos. ${ }^{17}$ The qualities of videos generated by the students in our study were of variable standards, but overall the videos were of acceptable quality. Inadequate sound clarity and editing were noticed in a few, and the students were advised to further edit the videos and to add subtitles to improve the quality.

We plan to further evaluate the time allotted for the activity to ensure adequate time for preparation and production of videos. Activity evaluation was based mainly on students' perception, which is a limitation in the current study. Further evaluation of the whole KOSE program through students' eventual performance in the clinic should also be considered. To enhance appreciation of the messages in watching the videos, there may be a need to provide some technical training in simple video production by experts in the field. Overall, however, the dental students in this study perceived the student-generated video activity as a positive learning experience.

\section{REFERENCES}

1. General Dental Council. The first five years: a framework for undergraduate dental education. 2nd ed. London: General Dental Council, 2002.

2. Alderson A, Martin M. Outcomes-based education: where has it come from and where is it going? Issues Educ Res 2007;17(2):161-82.

3. Klineberg I, Massey W, Thomas M, Cockrell D. A new era of dental education at the University of Sydney, Australia. Aust Dent J 2002;47(3):194-201.

4. Crean D. QuickTime streaming: a gateway to multi-modal social analyses. In: e-Xplore a face to face odyssey: proceedings of the Apple university consortium conference. Queensland, Australia: Apple Computer, 2001.

5. Ludewig A. iMovie: a student project with many side effects. In: e-Xplore a face to face odyssey: proceedings of the Apple university consortium conference. Queensland, Australia: Apple Computer, 2001.

6. Meeks M, Ilyasova A. A review of digital video production in postsecondary English classrooms at three universities. J Rhetoric Technol Pedagogy 2003;8(2). 
7. Shewbridge W, Berge ZL. The role of theory and technology in learning video production: the challenge of change. Int J E-learning 2004;3(1):9.

8. Learmont D. Collaborating for success: classroom teachers and video specialists. Cable in the Classroom. At: www. ciconline.org/Enrichment/Teaching/learningwithtechnology/magarticles/mag_0603_collaborating_for_success. htm 2003. Accessed: December 12, 2011.

9. Kolb DA, Fry R. Toward an applied theory of experiential learning. In: Kolb DA, Fry R, eds. Theories of group process. London: Wiley, 1975.

10. Knowles MS, Holton EF, Swanson RA. The adult learner: the definitive classic in adult education and human resource development. 6th ed. New York: Elsevier, 2005.

11. Gershen JA, Handelman SL. Role-playing as an educational technique in dentistry. J Dent Educ 1974;38(8): 451-5.

12. Kneebone R. Evaluating clinical simulations for learning procedural skills: a theory-based approach. Acad Med 2005;80(6):549.
13. Miller GE. The assessment of clinical skills/competence/ performance. Acad Med 1990;65(9):S63.

14. Lanning SK, Ranson SL, Willett RM. Communication skills instruction utilizing interdisciplinary peer teachers: program development and student perceptions. J Dent Educ 2008;72(2):172-82.

15. Students in the director's seat: teaching and learning with student-generated video. Proceedings of Ed-Media 2005 World Conference on Educational Multimedia, Hypermedia, and Telecommunications. Norfolk, VA: Association for the Advancement of Computing in Education, 2005.

16. Burn A, Brindley S, Durran J, Kelsall C, Sweetlove J, Tuohey C. "The rush of images": a research report into digital editing and the moving image. English in Educ 2001;35(2):34-48.

17. Schuck S, Kearney M. Capturing learning through studentgenerated digital video. Aust Educ Computing 2006;21:6. 